\title{
Food bioactives, micronutrients, immune function and COVID-19
}

\author{
Klaus W. Lange* and Yukiko Nakamura
}

Department of Experimental Psychology, University of Regensburg, Germany

*Corresponding author: Klaus W. Lange, Institute of Psychology, University of Regensburg, 93040 Regensburg, Germany. Tel: +49 941 9433815; Fax: +49 941 9434496; E-mail: klaus.lange@ur.de

DOI: $10.31665 /$ JFB.2020.10222

Received: June 22, 2020; Revised received \& accepted: June 28, 2020

Citation: Lange, K.W., and Nakamura, Y. (2020). Food bioactives, micronutrients, immune function and COVID-19. J. Food Bioact. 10: $1-8$.

\begin{abstract}
The development of targeted therapeutics against the novel coronavirus disease 2019 (COVID-19) is highly desirable but may present a challenge in the foreseeable future. Adequate nutrition is a prerequisite of an optimally functioning immune system. Nutritional approaches, including the administration of food bioactives and micronutrients, may therefore have the potential to augment immune function and defend against COVID-19. The supplementation of micronutrients, including vitamins and trace elements, and food bioactives, such as carotenoids and polyphenols, has shown itself to be beneficial in enhancing immunity in viral infections. However, the purported significance of these compounds in naturally occurring infections derives primarily from studies using animal models. The findings of human studies are inconsistent. The efficacy of micronutrients and food bioactives in infectious diseases can be affected by a wide array of factors, including the type of pathogen, the dose, timing and duration of supplementation and the characteristics of target populations. High-dose supplementation over extended periods of time may be associated with serious adverse effects, including aggravation of infectious diseases. Evidence evaluating dietary supplementation in COVID-19 is lacking. A reliance on supplements to prevent or treat COVID-19 would therefore be premature.
\end{abstract}

Keywords: Food bioactives; Micronutrients; Diet; Immune function; Coronavirus; COVID-19.

\section{Introduction}

In recent decades, the evolution of novel coronaviruses has resulted in an ongoing global threat to humans. Outbreaks of the severe acute respiratory syndrome (SARS) epidemic in 2002 and the Middle East respiratory syndrome (MERS) in 2015 were caused by highly pathogenic coronaviruses (De Wit et al., 2016; Yin and Wunderink, 2018). In December 2019, another previously unknown coronavirus, the severe acute respiratory syndrome coronavirus 2 (SARS$\mathrm{CoV}-2$ ), emerged, causing the novel infectious coronavirus disease 2019 (COVID-19), which has been declared a global pandemic by the World Health Organisation (WHO, 2020). Typical symptoms of COVID-19 include cough, fever, respiratory problems, gastrointestinal symptoms and, in severe cases, atypical pneumonia (Zhu et al., 2020). The cardiovascular, urinary and nervous systems may also be affected. Since the infection may be associated with mild symptoms or proceed asymptomatically, the number of undetected and unreported cases is likely to be high. The extent of the threat posed by the pandemic, in terms of infectiousness, virulence, need for medical care and case fatalities is currently unknown (Lange, 2020a; Lange, 2020b). Large-scale endeavours seeking to develop preventive and therapeutic strategies, including vaccination, antiviral agents and passive immunotherapy have been embarked upon. However, no drugs are currently known to shorten the duration, mitigate the severity or reduce the death rate of COVID-19. The efficacy of monoclonal antibody therapy also remains to be investigated. Moreover, the production, manufacture and global distribution of an effective and safe COVID-19 vaccine may require several years (Lange, 2020a). While the development of targeted therapeutics against COVID-19 is highly desirable, many problems remain to be solved and it may be unrealistic to expect effective prevention and treatment in the foreseeable future. 


\section{Immune functions}

The immune system is a highly complex biological system that has evolved to protect the host from various pathogens, such as bacteria, viruses, parasites and fungi as well as cancer cells, while tolerating non-threatening organisms, food and self (Parkin and Cohen, 2001). Immunity comprises two types of responses (innate and adaptive), which are involved in the identification and eradication of pathogens. Both innate and adaptive immunity involve cellular and humoral responses. The innate immune response depends, in part, on phagocytes and natural killer cells (Murphy and Weaver, 2016) and provides a fast, nonspecific response to pathogens without previous exposure or immunization. However, the speed and efficacy of innate immune responses do not increase in response to repeated exposure to pathogens. In contrast, adaptive, or acquired, immunity provides an immunological "memory" and is capable of generating an antigen-specific response to repeated infection with the same pathogen. The adaptive response involves antigenspecific cells (e.g. T lymphocytes), which coordinate the overall adaptive response or destroy virally-infected cells, and B lymphocytes, which can secrete antibodies (immunoglobulins) specific to the infecting pathogen (Murphy and Weaver, 2016). Induction of immunological memory is the mechanism underlying the protection provided by vaccines against subsequent pathogen exposure.

Nutrition is critically important for the immune system, with both malnutrition and overnutrition adversely affecting immune responses. The findings of epidemiological and clinical studies have shown that nutritional deficits may alter immune functions and increase the risk of infection (Chandra, 1991; Chandra, 1996; Watson, 1984). Infection with human immunodeficiency virus (HIV) increases the demand for micronutrients, such as essential trace minerals, while simultaneously causing a loss of these nutrients. The resulting deficiency can be compensated by supplementation of micronutrients (Bogden and Oleske, 2007; Stone et al., 2010).

The present viewpoint attempts to provide an overview of the evidence supporting a role of food bioactives and micronutrients in enhancing immune functions and will consider the obstacles needing to be overcome before recommendations regarding supplementation of micronutrients and food bioactives in the prevention and treatment of COVID-19 can be made.

\section{Micronutrients}

Nutrition is undoubtedly an important building block of immunity, and the influence of micronutrients on immune function has been widely investigated. Preservation of micronutrient homeostasis is a key factor in the maintenance of a healthy immune system, and a range of vitamins and trace elements have been shown to play an essential role in immune functions (Wintergerst et al., 2007). Deficiencies in micronutrients reduce immunity to disease while the supplementation of these compounds has been shown to enhance immunity in viral infections (Jayawardena et al., 2020).

Vitamins have long been known to influence immune functions, playing a role in both innate and adaptive immune responses (Mora et al., 2008). While the effects of some vitamins, such as vitamins $\mathrm{C}$ and $\mathrm{E}$ as well as $\mathrm{B}$ vitamins, are brought about in a relatively nonspecific manner (e.g. by anti-oxidative action), other vitamins, such as vitamins A and D, influence immune responses in highly specific ways (Mora et al., 2008). Reactive oxygen species are important in host defence and immunity (Rada and Leto, 2008). Viral infections, including those with HIV, hepatitis B and C viruses, Epstein-Barr virus, herpes simplex virus type 1 and influenza vi- ruses, frequently lead to the production of reactive oxygen species (Molteni et al., 2014). Oxidative stress is believed to play an important role in the infection process (Schreck et al., 1991). Furthermore, reactive oxygen species can enhance the replication of the HIV virus (Baruchel and Wainberg, 1992).

Vitamin A is a retinoid and a fat-soluble vitamin involved in the development of the immune system and in the regulation of cellular and humoral immune processes (Huang et al., 2018; Stephensen, 2001). A deficiency of vitamin A can cause impaired immunity and its supplementation has been shown to produce therapeutic effects in infectious diseases such as measles, pneumonia and contagious digestive diseases in children (Huang et al., 2018). While vitamin A supplementation does not appear to affect infection with the human immunodeficiency virus (HIV) per se, it may mitigate susceptibility to and reduce the incidence of other infectious diseases in individuals with HIV (Campa et al., 2017). Retinoids are molecules related to vitamin A that can increase and potentiate the actions of type 1 interferons, which are cytokines involved in the early innate immune response to viruses. The effects of retinoids combined with other antiviral agents could be assessed in pre-clinical SARS-CoV-2 studies (Trasino, 2020).

In addition to its well-established effects on calcium and bone homeostasis, vitamin D has often been suggested to be involved in decreasing the risk of microbial infection (Grant et al., 2020) by increasing physical barriers, cellular natural immunity, and adaptive immunity (Prietl et al., 2013; Rondanelli et al., 2018). An increase in susceptibility to immune-mediated disorders, such as chronic infections, has been linked in epidemiological studies to inadequate vitamin D concentrations (Baeke et al., 2010). Observational studies have found that vitamin D deficiency was associated with an increased risk of viral acute respiratory infection, and vitamin D supplementation has shown protective effects in a meta-analysis of clinical trials (Greiller and Martineau, 2015). However, the evidence in support of a role of vitamin D in the prevention of influenza infections is contentious (Gruber-Bzura, 2018).

A recent retrospective study assessed the plasma 25-hydroxyvitamin D levels in a cohort from Switzerland (D'Avolio et al., 2020). It was found that participants who were PCR-positive for SARS-CoV-2 $(\mathrm{N}=27)$ had statistically significantly lower 25-hydroxyvitamin $\mathrm{D}$ concentrations (median value $11.1 \mathrm{ng} / \mathrm{mL}$ ) than PCR-negative individuals ( $\mathrm{N}=80$, median $24.6 \mathrm{ng} / \mathrm{mL}$ ). This was also confirmed when the participants were stratified according to age $>70$ years (median values of $9.3[\mathrm{~N}=18]$ versus $23.1 \mathrm{ng} / \mathrm{mL}$ $[\mathrm{N}=43]$ ) (D'Avolio et al., 2020). These preliminary observations suggest that vitamin D supplementation might be useful in reducing the risk of SARS-CoV-2 infection. However, large population studies and randomized controlled trials need to confirm the findings.

Vitamin E is a lipid-soluble antioxidant that has been found to have immunomodulatory effects in cell-based, preclinical and clinical studies (Lee and Han, 2018; Lewis et al., 2019). Investigations in animal models have shown that vitamin $\mathrm{E}$ deficiency impairs immune functions, which can be corrected by vitamin E repletion (Wu and Meydani, 2019). However, the findings concerning the clinical significance of beneficial immune effects of vitamin $\mathrm{E}$ in humans, such as reducing the risk for respiratory infections, are inconsistent (Wu and Meydani, 2019)

Vitamin C (ascorbic acid) is a water soluble vitamin found in many foods, particularly citrus fruits and green vegetables. It is a potent antioxidant with anti-inflammatory and immune-supporting effects. Vitamin $\mathrm{C}$ accumulates at high levels in most immune cells and is involved in the immune defense through its support of various cellular functions of both the innate and adaptive immune systems (Ang et al., 2018). Although vitamin C supplementation 
is often claimed to exert preventive and therapeutic effects on the common cold (Bucher and White, 2016), a meta-analysis of available studies found no decrease in the incidence, severity or duration of the infection when vitamin $\mathrm{C}$ was administered as a daily supplement or at the onset of cold symptoms (Hemilä and Chalker, 2013). However, subgroup analysis found that regular vitamin C supplementation in people experiencing a high level of physical stress reduced the incidence of the common cold by $50 \%$ (Hemilä and Chalker, 2013). Vitamin C supplementation may be useful in the treatment of respiratory and systemic infections (Carr and Maggini, 2017). For example, the administration of vitamin $\mathrm{C}$ to people with acute respiratory infections appears to ameliorate the severity of respiratory symptoms (Hunt et al, 1994).

$B$ vitamins are water-soluble and act as components of coenzymes. They may mediate the interaction with immune cells involved in inflammation and various pathophysiological pathways (Spinas et al., 2015). B vitamin deficiency may weaken the host immune response (Yoshii et al., 2019).

Zinc is an essential trace element, whose role in immune function has been known for several decades and has been investigated extensively. Zinc is required for the release of vitamin A from the liver and its deficiency may lead to immune effects similar to those observed following vitamin A deficiency (Katona and Katona-Apte, 2008). Altered zinc homeostasis plays a crucial role in innate and adaptive immunity. The effects of zinc deficiency on immune cells can result in altered host defence, an increased inflammation risk and even death. Chronic zinc deficiency causes an increase in the production of pro-inflammatory cytokines, affecting the outcome of various inflammatory diseases (Bonaventura et al., 2014). Zinc supplementation can have anti-inflammatory and immunomodulatory effects and is capable of reversing the negative effects of zinc deficiency. Zinc can stimulate a wide range of signaling events, including antiviral responses (Maywald et al., 2017; Read et al., 2019). There is abundant evidence demonstrating effects of free zinc and zinc-binding proteins, such as metallothioneins, against various viruses, including measles and human immunodeficiency virus (HIV) (Gammoh and Rink, 2017; Read et al., 2019). The administration of zinc has the potential to significantly increase the clearance of both acute and chronic viral infections (Read et al., 2019). Zinc supplementation may improve the antiviral response and systemic immunity in zinc-deficient individuals and may also have the potential to specifically inhibit viral replication or symptoms related to infections (Read et al., 2019).

Selenium offers a wide array of health effects (Rayman, 2012). Dietary selenium levels and selenoprotein expression have been shown to impact the regulation of immune cell functions. Low selenium status has been found to be associated with poor immune functioning, while a higher selenium status promotes antiviral effects (Broome et al., 2004; Hoffmann, 2007). Selenium deficiency, which is the main regulator of selenoprotein expression, leads to impaired innate and adaptive immune responses (Avery and Hoffmann, 2018) and has been associated with the pathogenicity of several viruses (Guilin et al., 2019). A study in humans has demonstrated a functional outcome of selenium supplementation on the immune system. Individuals with low selenium status who received selenium supplementation and who were challenged with an orally administered, live, attenuated poliovirus cleared the virus more rapidly than placebo controls (Broome et al., 2004).

Iron and immunity are closely linked. Iron is an essential micronutrient for both humans and pathogenic microbes. Most human pathogens require iron, and the innate immune system is involved in limiting iron availability to invading microbes (Cassat and Skaar, 2013). Many aspects of the competition for iron between microorganisms and their hosts remain to be investigated (Ganz and Nemeth, 2015). Cross-regulatory interactions between iron homeostasis and immune function exist (Nairz et al., 2014). Alterations in iron homoeostasis, e.g. induced by pathological states involving iron loading or depletion, can adversely affect the ability of cells to respond to inflammation and infection (Ward et al. 2011). The interaction between iron status, iron supplementation and susceptibility to infection has been the subject of much debate, and the evidence is inconclusive. Supplementation of iron has been shown, for example, to decrease respiratory infection rates in children, while having deleterious effects on susceptibility to malaria (Oppenheimer, 2001). Iron supplementation may therefore be detrimental to immune function.

Copper plays an important role in the human immune system. It is involved in the functions of $\mathrm{T}$ helper cells, B cells, natural killer cells and macrophages (Percival, 1998). Dietary copper deficiency has been shown to affect both innate and adaptive immunity (Muñoz et al., 2007). Copper-deficient humans appear to have an increased susceptibility to infections, and copper may have the capacity to kill several infectious viruses, such as bronchitis virus, poliovirus and human immunodeficiency virus type 1 (Koller et al., 1987; Percival, 1998). It has been hypothesised that increasing plasma copper levels may have preventive and therapeutic effects against COVID-19 (Raha et al., 2020). This requires further investigation

Magnesium has been shown in animal models to play a role in both innate and acquired immune reponses (Galland, 1988). A compromised immune system may be found in elderly people, and a balanced magnesium homeostasis may be important in strengthening resistance to infection in the elderly (Tam et al., 2003). Suggestions have also been made that magnesium deficiency could contribute to the immunological alterations observed following strenuous exercise (Laires and Monteiro, 2008).

\section{Food bioactives}

Findings of epidemiological studies indicate that diets rich in carotenoids are associated with a lower incidence of infectious diseases, such as HIV infections (see Milani et al., 2017). Several studies have demonstrated the ability of dietary carotenes to prevent a wide range of infections in vitamin A-deficient rats (Green and Mallanby, 1930) and to reduce ear infections in children (Clausen, 1931). Since $\beta$-carotene exhibits provitamin A activity, the findings of these studies could be attributed to its conversion to vitamin A. However, the specific role of carotenoids can be investigated, for example, by administering carotenoids without provitamin A activity (e.g., lutein, lycopene, canthaxanthin, astaxanthin). Numerous investigations using non-provitamin A carotenoids have been able to show the immunomodulatory action of dietary carotenoids (Chew and Park, 2004). Non-provitamin A carotenoids were found to be as active or even more active than $\beta$-carotene in enhancing cellular and humoral immune responses in both experimental animals and humans (Chew and Park, 2004).

In addition to the well-established antioxidant properties of polyphenols, evidence suggests that they may be capable of modulating immune responses. The antiviral, antimicrobial, anti-inflammatory and cytotoxic capacity of flavonoids has been suggested to benefit the immune system (González-Gallego et al., 2010). Polyphenols have been demonstrated to influence the regulation of immune cells, the synthesis of pro-inflammatory cytokines and the suppression of pro-inflammatory gene expression (Yahfoufi et al., 2018).

Resveratrol (trans-3,5,4'-trihydroxy-stilbene) is a naturally oc- 
curring polyphenolic phytoalexin, which is present in the skin and seeds of various plants. Resveratrol administered at high doses has been found to activate adenosine monophosphate kinase, which is involved in the negative regulation of the NLRP3-inflammasome (Kulkarni and Cantó, 2015). The findings of experimental studies suggest a potential activity of resveratrol in a variety of human diseases, including viral infections such as acquired immunodeficiency syndrome (AIDS) and Middle East respiratory syndrome (MERS) (Lin et al., 2017; Malaguarnera, 2019). In a study assessing the antiviral properties of resveratrol against MERS-CoV, the compound was shown to significantly inhibit MERS-CoV infection and to prolong the survival of MERS-infected cells after infection (Lin et al., 2017). The expression of nucleocapsid protein essential for MERS-CoV replication was found to be reduced following the administration of resveratrol. In addition, resveratrol down-regulated the apoptosis induced by MERS-CoV in vitro (Lin et al., 2017).

Another mechanism underlying the antiviral activity of resveratrol may be the upregulation of the angiotensin-converting enzyme 2 (ACE2). ACE2 has been shown to be a functional SARS-CoV receptor in vitro (Li et al., 2003) and in vivo (Kuba et al., 2005) and is required for host cell entry and subsequent viral replication. SARS-CoV-2 also gains entry to the cell via the ACE2 receptor, with the viral trimeric spike protein of SARS-CoV-2 binding to the ACE2 receptor (Gheblawi et al., 2020; Yan et al., 2020). SARS$\mathrm{CoV}-2$ predominantly infects the lower respiratory tract through binding to ACE2 on alveolar epithelial cells (Jiang et al., 2020). While the levels of ACE2 expression appear to correlate with the susceptibility to SARS-CoV infection, the relationship with susceptibility to SARS-CoV-2 infection is unclear (Sommerstein et al., 2020). Upregulation of ACE2 has a protective effect on SARSCoV illness severity (Horne and Vohl, 2020). Thus, the ACE2 cellular receptor has been proposed as a key target of SARS-CoV treatment (Yu et al., 2018; Zhang et al., 2020). Dietary intake has been demonstrated to impact the expression and function of the ACE2 gene, with upregulation of ACE2 showing a protective effect on SARS-CoV illness severity (Yu et al., 2018). While a high intake of dietary fat may downregulate ACE2, high intake of resveratrol may upregulate ACE2 and play a protective role (Horne and Vohl, 2020).

The bioavailabllity of orally administered resveratrol is very low (Walle et al., 2004). The naturally dimethylated analogue of resveratrol, pterostilbene (trans-3,5-dimethoxy-4'hydroxystilbene) may be more effective due to its higher in vivo bioavailablity (Estrela et al. 2013; Walle et al., 2004). Stilbene derivatives may have antiviral effects against the SARS virus ( $\mathrm{Li}$ et al., 2006). Quercetin is a flavonol found in fruits and vegetables and has biological properties that may reduce the risk of infection ( $\mathrm{Li}$ et al., 2016). Quercetin has been shown to exhibit anti-inflammatory properties in cells from animals and humans and to ameliorate experimentally induced impairment of immunity function in animal models (Li et al., 2016). No effects on innate immune function or reduction of upper respiratory tract infection could be observed in humans (Li et al., 2016). The chronic supplementation of quercetin or flavonoid-rich foods appears to have minimal effects on immunity in humans (Peluso et al., 2015). However, a systematic review and meta-analysis assessing the effects of dietary flavonoids on upper respiratory tract infections found that flavonoids reduce the incidence of these infections when compared with controls, with no conclusive evidence regarding changes in duration or severity (Somerville et al., 2016). Furthermore, flavonoids stimulate natural killer cell activity and may therefore have some potential in the prevention of viral infections (Burkard et al., 2017). Potential immune boosting effects of polyphenols need to be established in future investigations with adequate scientific rigour.

Dietary fatty acids play a significant role in immune responses (Radzikowska et al., 2019). Omega-3 polyunsaturated fatty acids (PUFAs), in particular, are capable of altering the activation of cells of both the innate and the adaptive immune system (Gutiérrez et al., 2019). PUFAs have been shown in animal studies to exert powerful anti-inflammatory and immunomodulatory effects in a wide range of diseases (Fritsche, 2006). The impact of the administration of omega-3 PUFAs on the risk and outcome of infections is a matter of debate, since their intake may have both beneficial and deleterious effects in infectious diseases (Husson et al., 2016). The administration of eicosapentaenoic acid and docosahexaenoic acid in animals, corresponding to a daily dose of $500 \mathrm{mg}$ in humans, is beneficial against experimental infections caused by various pathogens. This effect may be attributed to the anti-inflammatory action of omega-3 PUFAs (Husson et al., 2016). However, omega-3 PUFAs administered at doses 2-to-4-fold higher or over extended periods of time may be detrimental in certain intestinal infections, since they may promote anti-inflammatory responses in people whose inflammatory response is critical for survival. High-dose and long-term supplementation of omega-3 PUFAs in humans should therefore be administered with great caution. Omega-3 PUFAs also appear to adversely affect the immune cell response in infections caused by intracellular pathogens such as mycobacterium tuberculosis, herpes simplex virus and influenza virus (Husson et al., 2016).

Specific probiotics, prebiotics or a combination of both exert significant effects on host immunological networks of the mucosal and systemic immune systems through their activation of multiple immune mechanisms (Frei et al., 2015; Maldonado Galdeano et al., 2019). Probiotics seem to have a supportive role in enhancing immune responses (Kang et al., 2013). Meta-analyses have reported modest efficacy of probiotics in reducing the incidence and duration of viral respiratory tract infections (Hao et al., 2015; King et al., 2014). Some patients with COVID-19 in China showed microbial dysbiosis with decreased Lactobacillus and Bifidobacterium (Xu et al., 2020). However, whether Lactobacilli and Bifidobacteria can support the balance of a diverse gut ecosystem in combating COVID-19 is unknown. The use of conventional probiotics for COVID-19 cannot be recommended before further studies reveal in more detail the effects of SARS-CoV-2 on gut microbiota (Mak et al., 2020).

Green tea and epigallocatechin-3-gallate appear to have multiple modulatory effects on innate and adaptive immunity (Pae and $\mathrm{Wu}, 2013)$. However, findings relevant to infectious diseases are not available.

\section{Problems surrounding the available findings}

Nutritional deficiency or inadequacy can impair immune functions, and optimal nutritional status is important in protecting against viral infections (Calder et al., 2020; Zhang and Liu, 2020) (see Table 1 ). Most evidence suggesting protective and other beneficial effects of micronutrients and food bioactives against infection stems from animal studies using various infection models. Ethical issues limit the use of experimental infections in humans. Human studies conducted to determine the impact of food compounds on naturally occurring infections are therefore observational.

A wide range of factors can affect the efficacy of micronutrients and food bioactives in infectious diseases. These factors include the type of pathogen, the dose, timing and duration of supplemen- 
Table 1. Potential involvement of micronutrients and food bioactives in viral infections

\begin{tabular}{ll}
\hline Agent & Virus \\
\hline Vitamin A & Measles virus, HIV, avian coronavirus \\
B vitamins & MERS-CoV \\
Vitamin C & Avian coronavirus; lower respiratory tract infections \\
Vitamin D & Viral acute respiratory infections; bovine coronavirus, SARS-CoV-2? \\
Vitamin E & Respiratory infections; coxsackievirus, bovine coronavirus \\
Selenium & Influenza virus, avian coronavirus, poliovirus \\
Zinc & Herpes simplex virus, HIV, measles virus, SARS-CoV? \\
Iron & Respiratory tract infections; viral mutations \\
Copper & Bronchitis virus, poliovirus, HIV \\
Carotenoids & HIV \\
Polyphenols & HIV, MERS-CoV \\
Omega-3 PUFAs & Herpes simplex virus, influenza virus (possibly harmful) \\
Probiotics & Viral respiratory tract infections \\
\hline
\end{tabular}

tation as well as characteristics of target populations (age, health status, nutritional status, immunological status, lifestyle, genetics).

Given the concerns surrounding the potential aggravation of infectious diseases associated with omega-3 PUFAs (Husson et al., 2016), the dosage of supplementation is important. A U-shaped relationship may exist between micronutrient status and health effects, as has been found, for example, regarding selenium intake (Bleys et al., 2008; Rayman, 2008). While additional intake derived from supplements may have benefits in individuals with low micronutrient status, those with adequate or high status may show adverse effects. Serious adverse outcomes may be associated with the administration of large amounts of micronutrients, such as selenium, anti-oxidative vitamins and omega-3 PUFAs (Klein et al., 2011; Lange et al., 2019; Miller 3rd et al., 2005; Rayman, 2008; Rutkowski and Grzegorczyk, 2012; Yang et al., 2012).

Distinct immune features can be observed in different stages of life, resulting in age-related differences in prevalence, type and severity of infections (Maggini et al., 2018). Numerous studies have presented evidence of decreasing immune function with ageing. Immunosenescence weakens the ability of elderly people to respond to infection and may be responsible for elevated morbidity and mortality from infectious diseases in the elderly (Gavazzi and Krause, 2002). Nutrition may carry the potential to delay or reverse age-related adverse changes in the immune system. However, different responses to nutritional interventions among different age groups should be considered. For example, ageing has been shown to alter the immune response to omega-3 PUFAs and probiotics (Yaqoob, 2017). Micronutrients that appear to be important in regard to immunosenescence include vitamin E (Wu and Meydani, 2014) and zinc (Mocchegiani et al., 2013). Enhancing immune functions with vitamin E may have significant clinical implications since its supplementation appears to be associated with improved resistance to influenza infection in aged mice and a decreased risk of upper respiratory infections in elderly humans (Wu and Meydani, 2014). Trials supplementing zinc in elderly people have shown contradictory effects on immunity (Mocchegiani et al., 2013).

A wide variety of dietary supplements, including "immunostimulants", antioxidant bioactives and anti-inflammatory micronutrients, are currently advertised as preventive measures against infections. Plausible arguments supporting the supplementation of micronutrients such as selenium or vitamin D derived mainly from cell culture or animal studies (Grant et al., 2020; Guilin et al., 2019). Recommendations regarding food bioactives such as polyphenols and carotenoids are based on similar experimental methods (Burkard et al., 2017; Chew and Park, 2004). However, no controlled human intervention studies have been conducted, and any benefits of nutrients in viral infections are therefore questionable. Even for HIV infection, with its increased demand for micronutrients, there are no meaningful clinical studies on the benefits of nutritional interventions (Tang et al., 2015). Furthermore, whether and, to what extent, there is an additional need for micronutrients or bioactives in other viral infections, including COVID-19 is unclear. A sufficient intake of food bioactives and micronutrients involved in immune function may be achieved through the consumption of a varied and well-balanced diet. However, nutrient deficiencies and inadequacies appear to be widespread (Bailey et al., 2015; Calder et al., 2020; Maggini et al., 2018).

\section{Conclusions}

Poor nutritional status predisposes to infections, and various micronutrients, including vitamins and trace elements, are essential for immunocompetence. Micronutrients and food bioactives can contribute in varying degrees to the maintenance of physical barriers to pathogens and to the two major types of immunity, innate and adaptive immunity. Nutritional immune support has the potential to augment viral defence. While the findings of animal studies confirm the importance of a wide range of food bioactives and micronutrients in immune functions, their specific roles in the prevention and treatment of viral infectious diseases, including COVID-19, in humans is far from clear. The lack of randomised controlled trials assessing the efficacy of food bioactives and micronutrients in the management of viral infections presents a major challenge in the evaluation of the role of these nutrients. Largescale epidemiological studies and well-designed clinical studies addressing dosage and combinations of these compounds in different populations are required to substantiate the benefits of supplementation in infection. If effective, micronutrient and bioactive 
Table 2. Major problems surrounding the use of food bioactives and micronutrients to boost immune function

Most evidence of protective effects of micronutrients and food bioactives against infection comes from animal studies

High-quality investigations on food bioactives and micronutrients, particularly randomised controlled trials, and immunity in humans are not available

Many factors can affect the efficacy of micronutrients and food bioactives in infectious diseases (type of pathogen; dosage, timing and duration of supplementation; age, health and nutritional status of target population)

Micronutrient and bioactive supplementation needs to be tailored to specific age-related needs

It is unclear whether and to what extent there is an additional need for micronutrients or bioactives in viral infections, including COVID-19

Prolonged supplementation of micronutrients and food bioactives at high doses may have adverse effects and may even aggravate infectious diseases

Evidence evaluating dietary supplementation in COVID-19 is lacking

supplementation would need to be tailored to specific age-related needs (see Table 2).

The experimental findings in animals and observations in humans suggesting a role of food bioactives and micronutrients in the prevention of viral infections including COVID-19 call for further studies. Evidence evaluating dietary supplementation in COVID-19 is lacking. Healthcare providers and patients should not therefore rely on supplements to prevent or treat COVID-19. A balanced diet containing diverse food bioactives and micronutrients may have supportive effects in COVID-19. At present, however, the most effective means of preventing the spread of the novel coronavirus is the avoidance of exposure to the virus by physical distancing and the wearing of facial masks and eye protection (Chu et al., 2020).

\section{References}

Ang, A., Pullar, J.M., Currie, M.J., and Vissers, M.C.M. (2018). Vitamin C and immune cell function in inflammation and cancer. Biochem. Soc. Trans. 46: 1147-1159.

Avery, J.C., and Hoffmann, P.R. (2018). Selenium, selenoproteins, and immunity. Nutrients 10: 1203

Baeke, F., Takiishi, T., Korf, H., Gysemans, C., and Mathieu, C. (2010). Vitamin D: modulator of the immune system. Curr. Opin. Pharmacol. 10: 482-496.

Bailey, R.L., West, K.P. Jr, and Black, R.E. (2015). The epidemiology of global micronutrient deficiencies. Ann. Nutr. Metab. 66: 22-33.

Baruchel, S., and Wainberg, M.A. (1992). The role of oxidative stress in disease progression in individuals infected by the human immunodeficiency virus. J. Leukoc. Biol. 52: 111-114.

Bleys, J., Navas-Acien, A., and Guallar, E. (2008). Serum selenium levels and all-cause, cancer, and cardiovascular mortality among US adults. Arch. Intern. Med. 168: 404-410.

Bogden, J.D., and Oleske, J.M. (2007). The essential trace minerals, immunity, and progression of HIV-1 infection. Nutr. Res. 27: 69-77.

Bonaventura, P., Benedetti, G., Albarede, F., and Miossec, P. (2014). Zinc and its role in immunity and inflammation. Autoimmun. Rev. 14: 277-285.

Broome, C.S., McArdle, F., Kyle, J.A., Andrews, F., Lowe, N.M., Hart, C.A., Arthur, J.R., and Jackson, M.J. (2004). An increase in selenium intake improves immune function and poliovirus handling in adults with marginal selenium status. Am. J. Clin. Nutr. 80: 154-162.

Bucher, A., and White, N. (2016). Vitamin C in the prevention and treatment of the common cold. Am. J. Lifestyle Med. 10: 181-183.

Burkard, M., Leischner, C., Lauer, U.M., Busch, C., Venturelli, S., and Frank, J. (2017). Dietary flavonoids and modulation of natural killer cell implications in cancer and viral diseases. J. Nutr. Biochem. 46: 1-12.

Calder, P.C., Carr, A.C., Gombart, A.F., and Eggersdorfer, M. (2020). Optimal nutritional status for a well-functioning immune system is an impor- tant factor to protect against viral infections. Nutrients 12: 1181

Campa, A., Baum, M.K., Bussmann, H., Sales Martinez, S., Farahani, M., van Widenfelt, E., Moyo, S., Makhema, J., Essex, M., and Marlink, R. (2017). The effect of micronutrient supplementation on active TB incidence early in HIV infection in Botswana. Nutr. Diet. Suppl. 37-45.

Carr, A.C., and Maggini, S. (2017). Vitamin C and immune function. Nutrients 9: 1211.

Cassat, J.E., and Skaar, E.P. (2013). Iron in infection and immunity. Cell Host Microbe 13: 509-519.

Chandra, R.K. (1991). 1990 McCollum Award Lecture. Nutrition and immunity: lessons from the past and new insights into the future. Am. J. Clin. Nutr. 53: 1087-1101.

Chandra, R.K. (1996). Nutrition, immunity and infection: from basic knowledge of dietary manipulation of immune responses to practical ap plication of ameliorating suffering and improving survival. Proc. Natl. Acad. Sci. U. S. A. 93: 14304-14307.

Chew, B.P., and Park, J.S. (2004). Carotenoid action on the immune response. J. Nutr. 134: 257S-261S.

Chu, D.K., Akl, E.A., Duda, S., Solo, K., Yaacoub, S., and Schünemann, H.J. (2020). Physical distancing, face masks, and eye protection to prevent person-to-person transmission of SARS-CoV-2 and COVID-19: a systematic review and meta-analysis. Lancet 395: 1973-1987.

Clausen, S.W. (1931). Carotinemia and resistance to infection. Trans. Am. Pediatr. Soc. 43: 27-30.

D’Avolio, A., Avataneo, V., Manca, A., Cusato, J., De Nicolò, A., Lucchini, R., Keller, F., and Cantù, M. (2020). 25-Hydroxyvitamin D concentrations are lower in patients with positive PCR for SARS-CoV-2. Nutrients 12: 1359.

De Wit, E., van Doremalen, N., Falzarano, D., and Munster, V.J. (2016). SARS and MERS: Recent insights into emerging coronaviruses. Nat. Rev. Microbiol. 14: 523-534.

Estrela, J.M., Ortega, A., Mena, S., Rodriguez, M.L., and Asensi, M. (2013). Pterostilbene: Biomedical applications. Crit. Rev. Clin. Lab. Sci. 50: 65-78.

Frei, R., Akdis, M., and O'Mahony, L. (2015). Prebiotics, probiotics, synbiotics, and the immune system: experimental data and clinical evidence. Curr. Opin. Gastroenterol. 31: 153-158.

Fritsche, K. (2006). Fatty acids as modulators of the immune response. Annu. Rev. Nutr. 26: 45-73.

Galland, L. (1988). Magnesium and immune function: an overview. Magnesium 7: 290-299.

Gammoh, N.Z., and Rink, L. (2017). Zinc in infection and inflammation. Nutrients 9: 624

Ganz, T., and Nemeth, E. (2015). Iron homeostasis in host defence and inflammation. Nat. Rev. Immunol. 15: 500-510.

Gavazzi, G., and Krause, K.H. (2002). Ageing and infection. Lancet Infect. Dis. 2: 659-666.

Gheblawi, M., Wang, K., Viveiros, A., Nguyen, Q., Zhong, J.-C., Turner, A.J., Raizada, M.K., Grant, M.B., and Oudit, G.Y. (2020). Angiotensin converting enzyme 2: SARS-CoV-2 receptor and regulator of the reninangiotensin system. Circ. Res. 126: 1456-1474.

González-Gallego, J., García-Mediavilla, M.V., Sánchez-Campos, S., and Tu- 
ñón, M.J. (2010). Fruit polyphenols, immunity and inflammation. Br. J. Nutr. 104(Suppl. 3): S15-27.

Grant, W.B., Lahore, H., McDonnell, S.L., Baggerly, C.A., French, C.B., Aliano, J.L., and Bhattoa, H.P. (2020). Evidence that vitamin D supplementation could reduce risk of influenza and COVID-19 infections and deaths. Nutrients 12: 988.

Green, H.N., and Mallanby, E. (1930). Carotene and vitamin A: the antiinfective action of carotene. Br. J. Exp. Pathol. 11: 81-89.

Greiller, C.L., and Martineau, A.R. Modulation of the immune response to respiratory viruses by vitamin D. Nutrients 7: 4240-4270.

Gruber-Bzura, B.M. (2018). Vitamin D and influenza-prevention or therapy? Int. J. Mol. Sci. 19: 2419.

Guilin, O.M., Vindry, C., Ohlmann, T., and Chavatte, L. (2019). Selenium, selenoproteins and viral Infection. Nutrients 11: 2101.

Gutiérrez, S., Svahn, S.L., and Johansson, M.E. (2019). Effects of omega-3 fatty acids on immune cells. Int. J. Mol. Sci. 20: 5028.

Hao, Q., Dong, B.R., and Wu, T. (2015). Probiotics for preventing acute upper respiratory tract infections. Cochrane Database Syst. Rev. 2015 CD006895.

Hemilä, H., and Chalker, E. (2013). Vitamin C for preventing and treating the common cold. Cochrane Database Syst. Rev. 2013: CD000980.

Hoffmann, P.R. (2007). Mechanisms by which selenium influences immune responses (Warsz). Arch. Immunol. Ther. Exp. 55: 289-297.

Horne, J.R., and Vohl, M.-H. (2020). Biological plausibility for interactions between dietary fat, resveratrol, ACE2, and SARS-CoV illness severity. Am. J. Physiol. Endocrinol. Metab. 318: E830-E833.

Huang, Z., Liu, Y., Qi, G, Brand, D., and Zheng, S.G. (2018). Role of vitamin $A$ in the immune system. J. Clin. Med. 7: 258.

Hunt, C., Chakravorty, N.K., Annan, G., Habibzadeh, N., and Schorah, C.J. (1994). The clinical effects of vitamin C supplementation in elderly hospitalised patients with acute respiratory infections. Int. J. Vitam. Nutr. Res. 64: 212-219.

Husson, M.O., Ley, D., Portal, C., Gottrand, M., Hueso, T., Desseyn, J.L., and Gottrand, F. (2016). Modulation of host defence against bacterial and viral infections by omega-3 polyunsaturated fatty acids. J. Infect. 73 : 523-535.

Jayawardena, R., Sooriyaarachchi, P., Chourdakis, M., Jeewandara, C., and Ranasinghe, P. (2020). Enhancing immunity in viral infections, with special emphasis on COVID-19: A review. Diabetes Metab. Syndr. 14: 367-382.

Jiang, F., Deng, L., Zhang, L., Cai, Y., Cheung, C.W., and Xia, Z. (2020). Review of the clinical characteristics of coronavirus disease 2019 (COVID-19). J. Gen. Intern. Med. 35: 1545-1549.

Kang, E.J., Kim, S.J., Hwang, I.H., and Ji, Y.J. (2013). The effect of probiotics on prevention of common cold: a meta-analysis of randomized controlled trial studies. Korean J. Fam. Med. 34: 2-10.

Katona, P., and Katona-Apte, J. (2008). The interaction between nutrition and infection. Clin. Infect. Dis. 46: 1582-1588.

King, S., Glanville, J., Sanders, M.E., Fitzgerald, A., and Varley, D. (2014). Effectiveness of probiotics on the duration of illness in healthy children and adults who develop common acute respiratory infectious conditions: a systematic review and meta-analysis. Br. J. Nutr. 112: 41-54.

Klein, E.A., Thompson, I.M.J., and Tangen, C.M. (2011). Vitamin E and the risk of prostate cancer: the Selenium and Vitamin E Cancer Prevention Trial (SELECT). JAMA 306: 1549-1556.

Koller, L.D., Mulhern, S.A., Frankel, N.C., Steven, M.G., and Williams, J.R. (1987). Immune dysfunction in rats fed a diet deficient in copper. Am. J. Clin. Nutr. 45: 997-1006.

Kuba, K., Imai, Y., Rao, S., Gao, H., Guo, F., Guan, B., Huan, Y., Yang, P., Zhang, Y., Deng, W., Bao, L., Zhang, B., Liu, G., Wang, Z., Chappell, M., Liu, Y., Zheng, D., Leibbrandt, A., Wada, T., Slutsky, A.S., Liu, D., Qin, C., Jiang, C., and Penninger, J.M. (2005). A crucial role of angiotensin converting enzyme 2 (ACE2) in SARS coronavirus-induced lung injury. Nat. Med. 11: 875-879.

Kulkarni, S.S., and Cantó, C. (2015). The molecular targets of resveratrol. Biochim. Biophys. Acta 1852: 1114-1123.

Laires, M.J., and Monteiro, C. (2008). Exercise, magnesium and immune function. Magnes. Res. 21: 92-96.

Lange, K.W. (2020a). The prevention of COVID-19 and the need for reliable data. Mov. Nutr. Health Dis. 4: 53-63.

Lange, K.W. (2020b). Mental health problems in COVID-19 and the need for reliable data. Mov. Nutr. Health Dis. 4: 64-69.

Lange, K.W., Nakamura, Y., Gosslau, A., and Li, S. (2019). Are there serious adverse effects of omega- 3 polyunsaturated fatty acid supplements? J. Food Bioact. 7: 1-6.

Lee, G.Y., and Han, S.M. (2018). The role of vitamin E in immunity. Nutrients 10: 1614.

Lewis, E.D., Meydani, S.N., and Wu, D. (2019). Regulatory role of vitamin $E$ in the immune system and inflammation. IUBMB Life 71: 487-494.

Li, W., Moore, M.J., Vasilieva, N., Sui, J., Wong, S.K., Berne, M.A., Somasundaran, M., Sullivan, J.L., Luzuriaga, K., Greenough, T.C., Choe, H., and Farzan, M. (2003). Angiotensin-converting enzyme 2 is a functional receptor for the SARS coronavirus. Nature 426: 450-454.

Li, Y., Yao, J., Han, C., Yang, J., Tabassum Chaudhry, M., Wang, S., Liu, H., and Yin, Y. (2016). Quercetin, inflammation and immunity. Nutrients 8: 167.

Li, Y.Q., Li, Z.L., Zhao, W.J., Wen, R.X., Meng, Q.W., and Zeng, Y. (2006). Synthesis of stilbene derivatives with inhibition of SARS coronavirus replication. Eur. J. Med. Chem. 41: 1084-1089.

Lin, S.C., Ho, C.T., Chuo, W.H., Li, S., Wang, T.T., and Lin, C.C. (2017). Effective inhibition of MERS-CoV infection by resveratrol. BMC Infect. Dis. 17: 144.

Maares, M., and Haase, H. (2016). Zinc and immunity: An essential interrelation. Arch. Biochem. Biophys. 611: 58-65.

Maggini, S., Pierre, A., and Calder, P. (2018). Immune function and micronutrient requirements change over the life course. Nutrients 10 1531.

Mak, J.W.Y., Chan, F.K.L., and Ng, S.C. (2020). Probiotics and COVID-19: one size does not fit all. Lancet Gastroenterol. Hepatol. 5: 644-645.

Malaguarnera, L. (2019). Influence of resveratrol on the immune response. Nutrients 11: 946.

Maldonado Galdeano, C., Cazorla, S.I., Lemme Dumit, J.M., Vélez, E., and Perdigón, G. (2019). Beneficial effects of probiotic consumption on the immune system. Ann. Nutr. Metab. 74: 115-124.

Maywald, M., Wessels, I., and Rink, L. (2017). Zinc signals and immunity. Int. J. Mol. Sci. 18: 2222.

Milani, A., Basirnejad, M., Shahbazi, S., and Bolhassani, A. (2017). Carotenoids: biochemistry, pharmacology and treatment. Br. J. Pharmacol. 174: 1290-1324.

Miller 3rd, E.R., Pastor-Barriuso, R., Dalal, D., Riemersma, R.A., Appel, L.J., and Guallar, E. (2005). Meta-analysis: high-dosage vitamin E supplementation may increase all-cause mortality. Ann. Intern. Med. 142: 37-46.

Mocchegiani, E., Romeo, J., Malavolta, M., Costarelli, L., Giacconi, R., Diaz, L.E., and Marcos, A. (2013). Zinc: dietary intake and impact of supplementation on immune function in elderly. Age 35: 839-860.

Molteni, C.G., Principi, N., and Esposito, S. (2014). Reactive oxygen and nitrogen species during viral infections. Free Radic. Res. 48: 11631169.

Mora, J.R., Iwata, M., and von Andrian, U.H. (2008). Vitamin effects on the immune system: vitamins $A$ and $D$ take centre stage. Nat. Rev. Immunol. 8: 685-698.

Muñoz, C., Rios, E., Olivos, J., Brunser, O., and Olivares, M. (2007). Iron, copper and immunocompetence. Br. J. Nutr. 98(Suppl. 1): S24-S28.

Murphy, K., and Weaver, C. (2016). Janeway's Immunobiology (9th ed). Garland Science/Taylor \& Francis, New York, NY.

Nairz, M., Haschka, D., Demetz, E., and Weiss, G. (2014). Iron at the interface of immunity and infection. Front. Pharmacol. 5: 152.

Oppenheimer, S.J. (2001). Iron and its relation to immunity and infectious disease. J. Nutr. 131: 616S-633S.

Pae, M., and Wu, D. (2013). Immunomodulating effects of epigallocatechin-3-gallate from green tea: mechanisms and applications. Food Funct. 4: 1287-1303.

Parkin, J., and Cohen, B. (2001). An overview of the immune system. Lancet 357: 1777-1789.

Peluso, I., Miglio, C., Morabito, G., loannone, F., and Serafini, M. (2015). Flavonoids and immune function in human: A systematic review. Crit. Rev. Food Sci. Nutr. 55: 383-395.

Percival, S.S. (1998). Copper and immunity. Am. J. Clin. Nutr. 67: 1064S$1068 \mathrm{~S}$.

Prietl, B., Treiber, G., Pieber, T.R., and Amrein, K. (2013). Vitamin D and immune function. Nutrients 5: 2502-2521. 
Rada, B., and Leto, T.L. (2008). Oxidative innate immune defenses by Nox/ Duox family NADPH oxidases. Contrib. Microbiol. 15: 164-187.

Radzikowska, U., Rinaldi, A.O., Celebi Sözener, Z., Karaguzel, D., Wojcik, M., Cypryk, K., Akdis, M., Akdis, C.A., and Sokolowska, M. (2019). The influence of dietary fatty acids on immune responses. Nutrients 11 : 2990.

Raha, S., Mallick, R., Basak, S., and Duttaroy, A.K. (2020). Is copper beneficial for COVID-19 patients? Med. Hypotheses 142: 109814.

Rayman, M.P. (2008). Food-chain selenium and human health: emphasis on intake. Br. J. Nutr. 100: 254-268.

Rayman, M.P. (2012). Selenium and human health. Lancet 379: 12561268.

Read, S.A., Obeid, S., Ahlenstiel, C., and Ahlenstiel, G. (2019). The role of zinc in antiviral immunity. Adv. Nutr. 10: 696-710.

Rondanelli, M., Miccono, A., Lamburghini, S., Avanzato, I., Riva, A., Allegrini, P., Faliva, M.A., Peroni, G., Nichetti, M., and Perna, S. (2018). Self-care for common colds: The pivotal role of vitamin $D$, vitamin $C$, zinc, and echinacea in three main immune interactive clusters (physical barriers, innate and adaptive immunity) involved during an episode of common colds-practical advice on dosages and on the time to take these nutrients/botanicals in order to prevent or treat common colds. Evid. Based Complement. Alternat. Med. 2018: 5813095.

Rutkowski, M., and Grzegorczyk, K. (2012). Adverse effects of antioxidative vitamins. Int. J. Occup. Med. Environ. Health 25: 105-121.

Schreck, R., Rieber, P., and Baeuerle, P.A. (1991). Reactive oxygen intermediates as apparently widely used messengers in the activation of the NF-kappa B transcription factor and HIV-1. EMBO J. 10: 2247-2258.

Somerville, V.S., Braakhuis, A.J., and Hopkins, W.G. (2016). Effect of flavonoids on upper respiratory tract infections and immune function: A systematic review and meta-analysis. Adv. Nutr. 7: 488-497.

Sommerstein, R., Kochen, M.M., Messerli, F.H., and Gräni, C. (2020). Coronavirus disease 2019 (COVID-19): Do angiotensin-converting enzyme inhibitors/angiotensin receptor blockers have a biphasic effect? J. Am. Heart Assoc. 9: e016509.

Spinas, E., Saggini, A., Kritas, S.K., Cerulli, G., Caraffa, A., Antinolfi, P., Pantalone, A., Frydas, A., Tei, M., Speziali, A., Saggini, R., Pandolfi, F., and Conti, P. (2015). Crosstalk between vitamin B and immunity. J. Biol. Regul. Homeost. Agents 29: 283-288.

Stephensen, C.B. (2001). Vitamin A, infection, and immune function. Annu. Rev. Nutr. 21: 167-192.

Stone, C.A., Kawai, K., Kupka, R., and Fawzi, W.W. (2010). Role of selenium in HIV infection. Nutr. Rev. 68: 671-681.

Tam, M., Gómez, S., González-Gross, M., and Marcos, A. (2003). Possible roles of magnesium on the immune system. Eur. J. Clin. Nutr. 57: 1193-1197.

Tang, A.M., Quick, T., Chung, M., and Wanke, C.A. (2015). Nutrition assessment, counseling and support interventions to improve healthrelated outcomes in people living with HIV/AIDS: a systematic review of the literature. J. Acquir. Immune Defic. Syndr. 68(Suppl. 3): S340S349.

Trasino, S.E. (2020). A role for retinoids in the treatment of COVID-19? Clin. Exp. Pharmacol. Physiol. doi:10.1111/1440-1681.13354.

Walle, T., Hsieh, F., DeLegge, M.H., Oatis Jr, J.E., and Walle, U.K. (2004). High absorption but very low bioavailability of oral resveratrol in humans. Drug Metab. Dispos. 32: 1377-1382.
Ward, R.J., Crichton, R.R., Taylor, D.L., Della Corte, L., Srai, S.K., and Dexter, D.T. (2011). Iron and the immune system. J. Neural Transm. 118: 315-328.

Watson, R.R. (Ed.). (1984). Nutrition, disease resistance, and immune function. Marcel Dekker, New York.

WHO. WHO. Rolling updates on coronavirus disease (COVID-19): WHO characterizes COVID-19 as a pandemic. Available: https://www.who. int/emergencies/diseases/novel-coronavirus-2019/events-as-theyhappen. Accessed March 30, 2020.

Wintergerst, E.S., Maggini, S., and Hornig, D.H. (2007). Contribution of selected vitamins and trace elements to immune function. Ann. Nutr Metab. 51: 301-323.

Wu, D., and Meydani, S.N. (2014). Age-associated changes in immune function: Impact of vitamin $E$ intervention and the underlying mechanisms. Endocr. Metab. Immune Disord. Drug Targets 14: 283-289.

Wu, D., and Meydani, S.N. (2019). Vitamin E, immune function, and protection against infection. In: Weber, P., Birringer, M., Blumberg, J.B., Eggersdorfer, M., and Frank, J. (Ed.). Vitamin E in human health. Humana Press, Cham, Switzerland, pp. 371-384.

Xu, K., Cai, H., Shen, Y., Ni, Q., Chen, Y., Hu, S., Li, J., Wang, H., Yu, L., Huang, H., Qiu, Y., Wei, G., Fang, Q., Zhou, J., Sheng, J., Liang, T., and Li, L. (2020). Management of corona virus disease-19 (COVID-19): the Zhejiang experience. Zhejiang Da Xue Xue Bao Yi Xue Ban 49(2): 147-157.

Yahfoufi, N., Alsadi, N., Jambi, M., and Matar, C. (2018). The immunomodulatory and anti-Inflammatory role of polyphenols. Nutrients 10 : 1618

Yan, R., Zhang, Y., Li, Y., Xia, L., Guo, Y., and Zhou, Q. (2020). Structural basis for the recognition of SARS-CoV-2 by full-length human ACE2. Science 367: 1444-1448.

Yang, C.S., Suh, N., and Kong, A.N.T. (2012). Does vitamin E prevent or promote cancer? Cancer Prev. Res. 5: 701-705.

Yaqoob, P. (2017). Ageing alters the impact of nutrition on immune function. Proc. Nutr. Soc. 76: 347-351.

Yin, Y., and Wunderink, R.G. (2018). MERS, SARS and other coronaviruses as causes of pneumonia. Respirology. 23: 130-137.

Yoshii, K., Hosomi, K., Sawane, K., and Kunisawa, J. (2019). Metabolism of dietary and microbial vitamin $B$ family in the regulation of host immunity. Front. Nutr. 6: 48.

Yu, H.-R., Tain, Y.-L., Tiao, M.-M., Chen, C.-C., Sheen, J-M., Lin, I.-C., Li, S.W., Tsai, C.C., Lin, Y.J., Hsieh, K.S., and Huang, L.T. (2018). Prenatal dexamethasone and postnatal high-fat diet have a synergistic effect of elevating blood pressure through a distinct programming mechanism of systemic and adipose renin-angiotensin systems. Lipids Health Dis. 17: 50.

Zhang, H., Penninger, J.M., Li, Y., Zhong, N., and Slutsky, A.S. (2020). Angiotensin-converting enzyme 2 (ACE2) as a SARS-CoV-2 receptor: molecular mechanisms and potential therapeutic target. Intensive Care Med. 46: 586-590.

Zhang, L., and Liu, Y. (2020). Potential interventions for novel coronavirus in China: A systematic review. J. Med. Virol. 92: 479-490.

Zhu, N., Zhang, D., Wang, W., Li, X., Yang, B., Song, J., Zhao, X., Huang, B., Shi, W., Lu, R., Niu, P., Zhan, F., Ma, X., Wang, D., Xu, W., Wu, G., Gao, G.F., Tan, W., and China Novel Coronavirus Investigating and Research Team (2020). A novel coronavirus from patients with pneumonia in China, 2019. N. Engl. J. Med. 382: 727-733. 